\title{
QUASICOMPACTNESS AND FUNCTIONALLY \\ HAUSDORFF SPACES
}

\author{
A. J. D'ARISTOTLE* \\ (Received 4 February 1970; revised 27 September 1971) \\ Communicated by B. Mond
}

\section{Introduction}

In [1] Van Est and Freudenthal introduced and studied several new separation axioms for a topological space. One of these was the $p \tau_{s} q$ axiom: Given distinct points $p$ and $q$ of $X$, there exists a real continuous function $f$ on $X$ with $f(p) \neq f(q)$. They observed that the $p \tau_{s} q$ axiom lies strictly between the Hausdorff and completely regular axioms, and that it neither implies nor is implied by the $T_{3}$ axiom. In [7] Proizvolov touched upon topological spaces with this property and called them functionally Hausdorff spaces.

The completely regular $T_{1}$ or Tychonoff spaces are precisely those topological spaces with Hausdorff compactifications. One of the best known compactifications of a Tychonoff space $X$ is the Stone-Cech compactification $\beta X$, and it is characterized as that compactification of $X$ in which $X$ is $C^{*}$ embedded [4]. A functionally Hausdorff space may fail to be regular, and so we cannot hope to embed an arbitrary functionally Hausdorff space in a compact functionally Hausdorff space. However, it is easily verified that corresponding to a functionally Hausdorff space $X$ there is a quasi compact functionally Hausdorff space $Y$ which contains $X$ as a dense, $C^{*}$ embedded subset. A topological $Y$ is said to be quasi compact if and only if every cover of $Y$ by cozero-sets of $Y$ has a finite subcover. Frolik defined and studied this concept in [3], where he also investigated other generalizations of compactness. In many situations quasicompactness rather than compactness is sufficient.

In this paper we continue the study of quasi-compactness and functionally Hausdorff spaces. In particular, we will show that there is a considerable amount of inter rlay between these two concepts.

"The author wishes to express his gratitude to Ralph Kopperman, O. Frink and the referees for their many helpful comments and suggestions. 


\section{Definitions}

Let $C(X)$ (or $C(X, \mathscr{T})$ ) be the set of all continuous, real-valued functions on a topological space $(X, \mathscr{T})$, and let $C^{*}(X)$ (or $C^{*}(X, \mathscr{T})$ ) denote the set of all bounded functions in $C(X)$. A subset $A$ of $X$ is said to be $C$-embedded in $X$ if every function in $C(A)$ can be extended to a function in $C(X)$. Similarly, the subset $A$ is said to be $C^{*}$-embedded in $X$ if every function in $C^{*}(A)$ can be extended to a function in $C^{*}(X)$.

A subset $A$ of a topological space $X$ is said to be a zero-set of $X$ if there exists a function $f$ in $C(X)$ such that

$$
A=f^{-1}(\{0\})=\{x \in X: f(x)=0\} .
$$

Complements of zero-sets are called cozero-sets.

A space $X$ is said to be completely regular if given any closed set $F$ and any point $x$ in its complement, there exists a function $f \in C(X)$ such that $f(x)=1$ and $f(y)=0$ if $y \in F$.

A thorough discussion of the previous definitions and the related theory may be found in [4].

\section{Remarks}

A topological space is quasi-compact if and only if every family of zero-sets of the space with the finite intersection property has a non-empty intersection. Any compact space is quasicompact, and a completely regular space is compact if and only if it is quasicompact. The continuous image of a quasicomfact space is quasicompact, but we have been unable to determine if a finite or arbitrary product of quasicompact spaces is quasicompact.

In the hypothesis of the Stone-Weierstrass Theorem, the underlying space $X$ is functionally Hausdorff and it is sufficient that this space be quasicompact rather than compact. To prove this, we modify the proof of this theorem given in [8] by replacing the word compact with quasicompact wherever it occurs.

Any quasicompact space is pseudocompact but there are examples of pseudocompact spaces which are not quasicompact. To see this we consider the space $W$ of all countable ordinals. We let $W(\alpha)$ denote the set of all ordinals less than a given ordinal $\alpha: W(\alpha)=\{\sigma: \sigma$ is an ordinal and $\sigma<\alpha\}$. We therefore have that $W=W\left(\omega_{1}\right)$ where $\omega_{1}$ is the first uncountable ordinal. The interested reader is referred to [4] for properties of these spaces.

In particular, we observe that every tail in $W$, that is, every set of the form $W-W(\alpha)$ where $\alpha$ is an ordinal less than $\omega_{1}$, is a zero-set of $W$, and that the family of all tails has the finite intersection property. Since $\omega_{1}$ has no immediate predecessor, the intersection of this family is empty, so that $W$ is not quasicompact. However, it is well-known that $W$ is pseudocompact. 
The following example, which we have obtained by modifying exercise 7 on page 86 of [6], shows that not every quasicompact space is compact. It is also an example of a non-regular functionally Hausdorff space. Let $I=[0,1]$ denote the unit interval with the usual topology $\mathscr{T}_{1}$, and let $H$ be the set of all rational numbers in the open interval $\left(\frac{1}{2}, 1\right)$. Consider the space $\left(I, \mathscr{T}_{2}\right)$ where $\mathscr{T}_{2}$ is the topology on $I$ generated by $\mathscr{T}_{1} \cup\{H\}$ as a subbase. Since $\mathscr{T}_{1} \subset \mathscr{T}_{2}$, it follows that $\left(I, \mathscr{T}_{2}\right)$ is a functionally Hausdorff space. However, $\left(I, \mathscr{T}_{2}\right)$ is not regular. The point $\frac{3}{4}$ is a member of the open set $H$, but there is no open set containing this point whose closure is contained in $H$.

It is easily verified that $\left(I, \mathscr{T}_{1}\right)$ and $\left(I, \mathscr{T}_{2}\right)$ have the same real-valued continuous functions and hence the same cozero-sets. Since $\left(I, \mathscr{T}_{1}\right)$ is compact, it follows that $\left(I, \mathscr{T}_{2}\right)$ is quasi-compact. However, $\left(I, \mathscr{T}_{2}\right)$ is not compact, since a compact $T_{2}$ space is regular.

The functionally Hausdorff property is both hereditary and productive. In [2] Frink provided an internal characterization of a Tychonoff space. A $T_{1}$ space $X$ is Tychonoff if and only if it has a normal base. For functionally Hausdorfl spaces the situation is much simpler. A space $X$ is functionally Hausdorff if and only if the family of zero-sets of $X$ separates points.

THEOREM 1. A functionally Hausdorff space $\left(X, \mathscr{T}_{1}\right)$ can be densely $C^{*}$ embedded in a quasicompact functionally Hausdorff space $Y$.

ProOF. Let $\mathscr{T}_{2}$ be the topology on $X$ generated by the cozero-sets of $\mathscr{T}_{1}$ as a base for the open sets. It is easily verified that $\left(X, \mathscr{T}_{2}\right)$ is a Tychonofl space and that $C\left(X, \mathscr{T}_{1}\right)=C\left(X, \mathscr{T}_{2}\right)$. If $\left(Y, \mathscr{T}_{3}\right)$ is the Stone-Cech compactification of $\left(X, \mathscr{T}_{2}\right)$, let $\mathscr{T}_{4}$ be the topology on $Y$ generated by $\mathscr{T}_{1} \cup \mathscr{T}_{3}$ as a subbase. Clearly $\left(X, \mathscr{T}_{1}\right)$ is a $C^{*}$-embedded in $\left(Y, \mathscr{T}_{4}\right)$.

If $A$ is a zero-set of $\left(Y, \mathscr{T}_{4}\right)$, then we may write $A=f^{-1}(\{0\})$ where $f \in C^{*}\left(Y, \mathscr{T}_{4}\right)$. Now $f \mid X \in C^{*}\left(X, \mathscr{T}_{1}\right)=C^{*}\left(X, \mathscr{T}_{2}\right)$ and so there is a function $g \in C\left(Y, \mathscr{T}_{3}\right)$ which agrees with $f$ on $X$. Since $f$ and $g$ are members of $C\left(Y, \mathscr{T}_{4}\right)$ and $X$ is dense in $Y$, we have that $f=g$ (see Exercise 3C of [5]). It is now clear that $\left(Y, \mathscr{T}_{3}\right)$ and $\left(Y, \mathscr{T}_{4}\right)$ have the same zero-sets which implies that $\left(Y, \mathscr{T}_{4}\right)$ is quasicompact.

A Hausdorff space is absolutely closed if and only if it is closed in every Hausdorff space in which it can be embedded. This notion is a generalization of a property of compact Hausdorff spaces. There is an extensive literature associated with absolutely closed spaces, and a number of references may be found on page 304 of [9]. It is natural to inquire about the analogue of this condition for functionally Hausdorff spaces.

THEOREM 2. A functionally Hausdorff space $X$ is closed in every functionally Hausdorff space in which it can be embedded if and only if it is quasicompact. 
Proof. Suppose the functionally Hausdorff space $X$ is quasicompact and that it is embedded in a functionally Hausdorff space $Y$. Then if $y \in Y-X$ there is for each $p \in X$ a real-valued continuous function $f_{p}$ on $Y$ with $f_{p}(p)=0$ and $f_{p}(y)=1$. It is clear that $C_{p}=\left\{s \in Y: f_{p}(s)<\frac{1}{2}\right\} \cap X$ is a cozero-set of $X$ for each $p$ in $X$, and that $X=\bigcup_{p \in X} C_{p}$. But $X$ is quasicompact and so there exist elements $p_{1}, p_{2}, \cdots, p_{n}$ of $X$ with $X=\bigcup_{i=1}^{n} C_{p_{i}}=\bigcup_{i=1}^{n}\left\{s \in Y ; f_{p_{i}}(s)<\frac{1}{2}\right\}$. We then have $y$ a member of $\bigcap_{i=1}^{n}\left\{s \in Y: f_{p_{i}}(s)>\frac{1}{2}\right\}$ which is seen to be an open set of $Y$ disjoint from $X$. It follows that $X$ is a closed subset of $Y$.

Conversely, if the functionally Hausdorff space $X$ is not quasicompact, then by Theorem $1, X$ is a dense subset of a quasicompact functionally Hausdorff space $Y$. Now $Y$ is quasicompact which implies that $X$ cannot be closed in $Y$.

We now investigate how the notion of quasicompactness may be related to a subset of a topological space. We presently show that quasicompactness, unlike compactness, is not an absolute property.

Definitions. A subset $A$ of a topological space $X$ is said to be quasicompact if $A$ with the induced topology is quasicc mpact; $A$ is said to be externally quasicompact relative to $X$ is every cover of $A$ by cozero-sets of $X$ has a finite subcover.

The subsets $A$ and $B$ of a space $X$ are said to be completely separated in $X$ if there exists a function $f$ in $C^{*}(X)$ such that $f(x)=0$ for all $x$ in $A$, and $f(x)=1$ for all $x$ in $B$.

Although every quasicompact subset of a topological space $X$ is externally quasicompact relative to $X$, the following example shows that even in a functionally Hausdorff space a subset which is externally quasicompact relative to $X$ need not te quasicompact.

We modify the example given by Gillman and Jerison in Chapter 3 of [4]. Let

$$
I^{2}=\{(x, y): 0 \leqq x \leqq 1,0 \leqq y \leqq 1\}
$$

be the unit square with the usual to ${ }^{-} \operatorname{ology} \mathscr{T}_{1}$ and let

$$
A=\left\{(x, 0):(x, 0) \in I^{2}\right\} \text {. }
$$

To each $(x, 0) \in A$ define

$$
V_{x}=\{(x, 0)\} \cup\left\{(u, v) \in I^{2}: v>0 \text { and }(u-x)^{2}+v^{2}<\left(\frac{1}{4}\right)^{2}\right\} .
$$

Let $\mathscr{T}_{2}$ be the topology on $I^{2}$ generated by the collection of sets $\mathscr{T}_{1} \cup\left(V_{x}\right)_{x \in I}$ as a subbase. It is easily verified that $\left(I^{2}, \mathscr{T}_{1}\right)$ and $\left(I^{2}, \mathscr{T}_{2}\right)$ have the same continuous functions. This implies that $A$ is externally quasicompact relative to the functionally Hausdorff space $\left(I^{2}, \mathscr{T}_{2}\right)$. But $A$ with the topology irduced by $\mathscr{T}_{2}$ is a ciscrete space and hence is not quasicompact. 
Let $\left(X, \mathscr{T}_{1}\right)$ be a functionally Hausdorff space, and suppose $A$ is a subset of $X$ which is externally quasicompact relative to $X$. If $B$ is an intersection of zero-sets of $X$ (note $\{p\}$ is such a set for each $p$ in $X$ ) and $A \cap B=\phi$, then $A$ and $B$ are completely separated. This generalizes and follows from the wellrecognized fact that in a completely regular space, any two disjoint closed sets, one of which is compact, are completely sep rated $\lfloor 4]$. We ne.d merely consider the Tychonoff topology $\mathscr{T}_{2}$ on $X$ generated by the cozero-sets of $\mathscr{T}_{1}$.

It is well-known that a compact subset of a Tychonoff space is $C$ embedded [4]. The following theorem is an improvement of that result.

THEOREM 3. Every quasicompact subset of a functionally Haus.lorff space is C-embedded.

PROOF. Suppose $A$ is a quasicompact subset of a functionally Hausdorff space $(X, \mathscr{T})$ so that $\left(A, \mathscr{T}_{1}\right)$ is a quasicompact topological space where $\mathscr{T}_{1}$ is the induced topology. Let $\mathscr{T}_{2}$ and $\mathscr{T}_{3}$ be the topologies on $X$ and $A$ respectively, generated by the cozero-sets of $(X, \mathscr{T})$ and $\left(A, \mathscr{T}_{1}\right)$. Furthermore, let $\mathscr{T}_{4}$ be the topology on $A$ induced by $\mathscr{T}_{2}$. A base for $\mathscr{T}_{4}$ consists of all sets of the form $C \cap A$ where $C$ is a cozero-set of $(X, \mathscr{T})$. Each such basic element of $\mathscr{T}_{4}$ is a cozero-set of $\left(A, \mathscr{T}_{1}\right)$ and thus is a member of $\mathscr{T}_{3}$. It is now clear that $\mathscr{T}_{4} \subset \mathscr{T}_{3}$. The basic open sets of $\left(A, \mathscr{T}_{3}\right)$ are the cozero-sets of $\left(A, \mathscr{T}_{1}\right)$ and so $\left(A, \mathscr{T}_{1}\right)$ being quasicompact implies that $\left(A, \mathscr{T}_{3}\right)$ is compact. It follows that the identity mapping $i:\left(A, \mathscr{T}_{3}\right) \rightarrow\left(A, \mathscr{T}_{4}\right)$ is a home omorphism because it is a $1-1$, continuous map from a compact space onto a Hausdorff space. Thus, $\mathscr{T}_{3}=\mathscr{T}_{4}$.

If $g \in C\left(A, \mathscr{T}_{1}\right)$ it follows that $g \in C\left(A, \mathscr{T}_{3}\right)=C\left(A, \mathscr{T}_{4}\right)$. But each compact subset of a Tychonoff space is $C$-embedded, and so $\mathrm{t}_{\text {. }}$ ere exists a function $h \in C\left(X, \mathscr{T}_{2}\right)$ which agrees with $g$ on $A$. Now $h \in C(X, \mathscr{T})$ and this completes the proof of the theorem.

However, the above result does not hold if $A$ is merely externally quasicompact relative to $X$. To see this we refer to the unit square example which precedes the above theorem. The set $A$ with the topology induced by $\mathscr{T}_{2}$ is a discrete space, and no unbounded real-valued function on $A$ will have continuous extension to the pseudocompact space $\left(I^{2}, \mathscr{T}_{2}\right)$. Hence $A$ is not $C$-embedied.

In problem $5 \mathrm{~B}$ of [5], it is observed that the closure of a compact subset of a topological space may fail to be comp.ct. However, in the case of quasicompactness we have the following result.

THEOREM 4. Let $A$ be a subset of a topological space $X$. If $A$ is quasicompact (externally quasicompact relative to $X$ ), then the closure of $A$ is quasicompact (externally quasicompact relative to $X$ ).

Proof. Suppose that $A$ is a quasicompact subset of $X$, and that $\left\{C_{\lambda}\right\}_{\lambda \subseteq \Lambda}$ 
is a family of cozero-sets in $\mathrm{cl}_{X} A$ whose union is $\mathrm{cl}_{X} A$. If $p \in A$, then $p \in C_{\lambda_{p}}$ for some $\lambda_{p} \in \Lambda$, and so there is a cozero-set $D_{\lambda_{p}}$ of $\mathrm{cl}_{X} A$ such that

$$
p \in D_{\lambda_{p}} \subseteq \mathrm{cl}_{c l X} A D_{\lambda_{p}} \subseteq C_{\lambda_{p}} \text {. }
$$

We note that $\operatorname{cl}_{\operatorname{cl}_{X} A} D_{\lambda_{p}}=\operatorname{cl}_{X} D_{\lambda_{p}}$. Now $A \subseteq \bigcup_{p \in A} D_{\lambda_{p}}$ and since $A$ is quasicompact, there exist $p_{1}, p_{2}, \cdots, p_{n} \in A$ such that $A \subseteq \bigcup_{i=1} D_{\lambda_{p i}}$. Therefore,

$$
\mathrm{cl}_{X} A \subseteq \bigcup_{i=1}^{n} \mathrm{cl}_{X} D_{\lambda_{p i}} \subseteq \bigcup_{i=1}^{n} C_{\lambda_{p i}}
$$

and hence $\operatorname{cl}_{X} A$ is quasicompact. The proof for subsets which are externally quasicompact relative to $X$ is similar.

However, the unit square example shows that the closure of a subset which is externally quasicompact relative to $X$ need not be quasicompact. We now give a sufficient condition for a $T_{1}$ space to be a functionally Hausdorff space.

THEOREM 5. If every finite subset of a $T_{1}$ space $X$ is $C$-embedded, then $X$ is a functionally Hausdorff space.

Proof. If $p, q \in X$ with $p \neq q$, then $X$ being a $T_{1}$ space implies that the set $\{p, q\}$ has the discrete topology as a subspace of $X$. The continuous function $g$ from $\{p, q\}$ to $R$ defined by $g(p)=0$ and $g(q)=1$ can be continuously extended to $X$. Hence, $X$ is a functionally Hausdorff space.

COROLLARY. If every subset which is externally quasicompact relative to $X$ (every quasicompact subset) is $C$-embedded, then $X$ is a functionally Hausdorff space.

\section{References}

[1] W. T. Van Est and H. Freudenthal, 'Trennung durch statige funktionen in topologischen räumen', Indag. Math. 15 (1951), 359-368.

[2] Orrin Frink, 'Compactifications and semi-normal spaces,' Amer. J. Math. 86 (1964), 602-607,

[3] Z. Frolik, 'Generalizations of compact and Lindelöf spaces,' Czech. Math. J. 9 (84), (1959), 172-217.

[4] L. Gillman and M. Jerison, Rings of continuous functions (Van Nostrand, New York, 1960).

[5] J. L. Kelley, General topology (Van Nostrand, New York, 1955).

[6] W. J. Pervin, Foundations of general topology (Academic Press, New York, 1964).

[7] V. V. Proizvolov, 'On one-to-one continuous mappings of topological spaces,' Mat. Sb. (N. S.) 60 (110), (1965), 417-431.

[8] W. Rudin, Principles of mathematical analysis (McGraw-Hill, New York, 1964).

[9] S. Willard, General topology (Addison-Wesley, Reading, Massachusetts, 1970).

Brooklyn College of the City University of New York

State University College at Geneseo

U.S.A. 